Menschenbilder - Gesundheitsbilder: Beispiele ihres Verhältnisses aus der Antike und deren Bedeutung für die Gegenwart

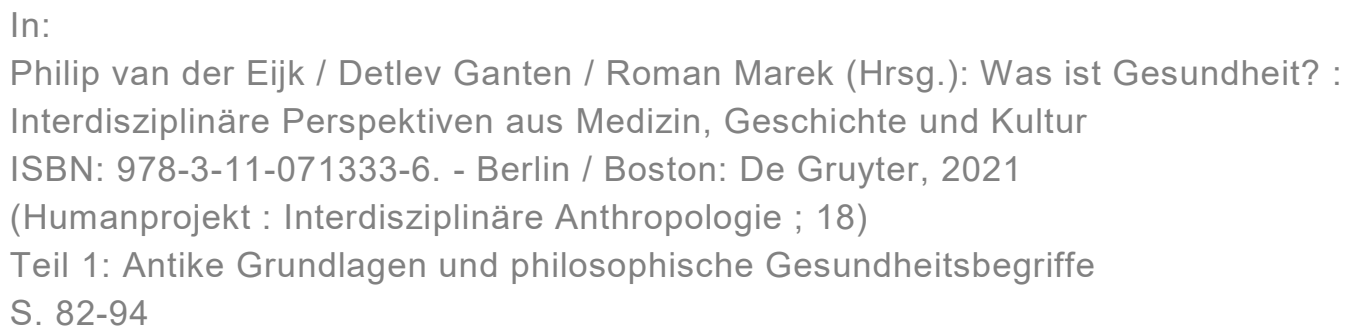




\title{
Menschenbilder - Gesundheitsbilder: Beispiele ihres Verhältnisses aus der Antike und deren Bedeutung für die Gegenwart
}

\begin{abstract}
Concepts of Man - Concepts of Health: A Glimpse of Their Relationship in Antiquity With Relevance to Our Day and Age. Referring to ancient miraculous healing narratives, this article argues that concepts of health are inextricably intertwined with concepts of man. However, the relatively autonomous idea of medical treatments based on scientific reasoning is not an invention of modern secularization. It already existed in antiquity - even among people of faith. Gods and other religious authorities were regarded as mediating factors; they were not held responsible for diseases or cures. Examples from Christian and pagan traditions show that the interplay between ideas of man and concepts of health were extremely complex and diverse. Obviously, this was true already in antiquity - but it is even more evident in the present. Dualistic confrontations (e.g., pre-modern versus modern times, pre-scientific healing vs. academic medicine) are of little help to achieve universal health care and global health.
\end{abstract}

Dass Menschen- und Gesundheitsbilder eng aufeinander bezogen sind, ist zunächst einmal eine vergleichsweise triviale Einsicht. Sie verführt meiner Ansicht nach aber immer wieder dazu, schlichte Duale zu modellieren und einlinige Kausalitäten zu formulieren - beispielsweise in der Gegenüberstellung von Moderne und Vormoderne, wissenschaftlichem und religiösem Menschenbild und so weiter und so fort. Prekär und nicht einfach zu beschreiben ist offenbar die exakte Verbindung zwischen Menschen- und Gesundheitsbildern, die im Titel nur durch einen Gedankenstrich markiert ist, aber natürlich durch die Kopula „und“ verdeutlicht werden könnte: Menschen- und Gesundheitsbilder. Mir kommt es hier darauf an, solche vertrauten und scheinbar klaren Differenzierungen zwischen Antike und Gegenwart oder einem religiösen und einem wissenschaftlichen Zugriff im Blick auf Menschen- und Gesundheitsbilder ein wenig zu perforieren, mindestens festgefügte Grenzen zu perforieren. Ich tue das an scheinbar (zumindest für Menschen, die sich nicht hauptberuflich mit der paganen und christlichen Antike beschäftigen) marginalen Texten. Der Anspruch ist aber,

Christoph Markschies, Berlin-Brandenburgische Akademie der Wissenschaften; Lehrstuhl für Antikes Christentum, Theologische Fakultät, Humboldt-Universität zu Berlin

Ә OpenAccess. ๑ 2021 Christoph Markschies, publiziert von De Gruyter. [(c) Bry-Nc-SA Dieses Werk ist lizenziert unter einer Creative Commons Namensnennung - Nicht kommerziell - Weitergabe unter gleichen Bedingungen 4.0 International Lizenz. https://doi.org/10.1515/9783110713336-008 
durch den Rückblick in die Vergangenheit auch zu konturieren, was für die Gegenwart Bedeutung hat. Schließlich geht es bei der Frage nach holistischen Ansätzen immer auch um eben diesen Zusammenhang von Menschen- und Gesundheitsbildern (Thumiger 2020).

$\mathrm{Zu}$ diesem Zweck setze ich zunächst bei einer antiken christlichen Textpassage ein, die einer der verschiedenen Versionen der Vita der Arzt-Heiligen Cosmas und Damian entstammt und überschrieben ist mit „Über den Bauern mit dem verfaulten Fuß“. Ich tue das, weil ich mich in einem Forschungsprojekt einige Jahre mit dem Zusammenhang zwischen Heil und Heilung in der christlichen Antike beschäftigt habe (Markschies 2008), dazu auch ein wenig veröffentlicht habe und Analysen, die bei konkreten Texten ihren Ausgang nehmen, ohnehin sehr schätze (Markschies 2006a, 2006b). Das Beispiel stammt aus dem Kontext christlicher Erzählungen über heilige, nach damaligen Maßstäben also besondere und wundertätige Menschen. Die (etwas längere) Passage lautet:

Es war ein Bauer, der allzeit ein unzüchtiges Leben hatte. Denn obwohl er im Gegenteil zu dem, was Salomon sagt, ,,jeder Faule ist in seinen Begierden ,verhaftet““, ${ }^{1}$ nicht faul war, hielt er sich an verderbliche Praktiken und lebte mit Vergnügungssüchtigen zusammen. Was er aus der landwirtschaftlichen Arbeit erwirtschaftete, gab er für Huren aus und gab sich immerfort und unersättlich mit jenen der Zügellosigkeit hin. Aber auch nichts anderes in seinem Handeln war von guter Art - Gott zu erzürnen war er immerzu beschäftigt. Als er nun einmal mit einer Hacke die Erde wie gewohnt umgrub, verletzte er sich mit dieser sein rechtes Bein und fügte sich dadurch eine tiefe Wunde zu. Von seinen Mitarbeitern getragen, wurde er ins Haus gebracht und hingelegt, weil er meinte, ausschließlich von der Wunde geheilt werden zu müssen. Als es aber Nacht geworden war, schwoll die Wunde so stark an, dass sie sich an Umfang vom gesamten Körper abhob. Und mit Tagesanbruch rufen die Anwesenden einen Arzt, der, als er sieht, dass das Leiden ernst ist und bald von Blutvergiftung befallen sein würde, mahnend sagt: „Wenn du unter den Lebenden weilen und nicht augenblicklich sterben willst, musst du mir gestatten, das kranke Bein zu amputieren. Denn wenn ich es nicht bald abschneide, wird sich das Leiden über den ganzen Körper ausbreiten und auf schnellstem Weg den Tod herbeiführen“. Nachdem der Arzt ihm solches angezeigt hatte, ging er fort, um das für die Amputation notwendige Gerät und das (Brenn-)Eisen zu besorgen. Wie nun dem Bauern all das Schlechte in den Sinn kam, sobald er sich von seiner Pein sammelte, begann er zu weinen und zu seufzen und sagte: „Herr und Gott! Du allein kennst meine Sünden, dass sie nicht zu erzählen sind. Aber auch ich kenne Dein Erbarmen, das Deiner Großherzigkeit gemäß ist, sowie auch Dein Mitleid. Menschenhände taugen nicht für mein Leiden, sondern Du heile mich durch Deine Diener Kosmas und Damian, denen Du die Gnade der schmerzlosen Heilung schenkst“. Als er mit diesen und ähnlichen Worten geklagt und Gott angefleht hatte, erschienen ihm um die Mitternacht die Heiligen in Gestalt von Ärzten und sprachen: „O Menschenkind, den dir der Erziehung wegen zugefügten vorübergehenden Schmerz konntest du nicht ertragen, wie willst du die für dich bereitete

1 Kohelet 13,4, vgl. auch 10,4. 
ewige Strafe bestehen? Doch wenn du dort und hier gerettet werden willst, so bereue und beschließe es mit uns durch einen Eid: dass du den Rest deines Lebens besonnen verbringen und auch alle anderen Gesetze Gottes erfüllen wirst!“ Und er schwor es mit Furcht und größter Freude und schloss den Pakt, denn er erkannte, dass sie die Heiligen waren. Nun hoben sie den verwundeten Fuß in die Höhe und drückten den Eiter ganz heraus, reinigten die Wunde und heilten sie allein durch Berührung, so dass keinerlei Narbe an ihm zu erkennen war. Und sie sagten zu ihm: „Siehe, kein Übel ist an deinem Körper. Heile auch deine Seele!“ Und mit diesen Worten gingen sie von ihm fort. Am folgenden Tag stand der Bauer sehr früh auf und ging auf sein Feld, um das Gewohnte zu erledigen, voll Freude über die Rettung seiner Seele und seines Körpers. Er verbrachte nämlich den Rest seines Lebens mit den tugendhaftesten Handlungen. Als die Sonne aufgegangen war, kam der Arzt und brachte die Amputationsgeräte. Als er hörte, dass jener kein Übel mehr hatte, machte er sich auf den Weg zu dem Feld und wurde, weil er in der ganzen Gegend herumlief, zum Botschafter des Wunders. Er selbst und der Geheilte und alle, die von dem Geschehen hörten, lobten Gott, der seinen Heiligen eine solche Gnade gewährt (Rupprecht 1935, S. 15,5-17,16 [miracu$\operatorname{lum} 4])^{2}$

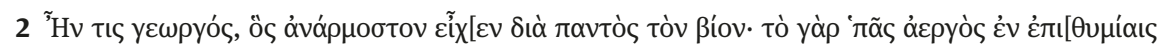

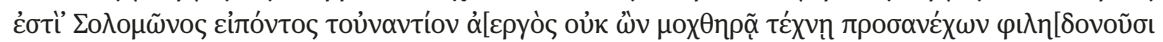

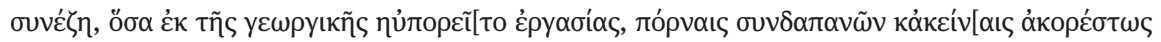

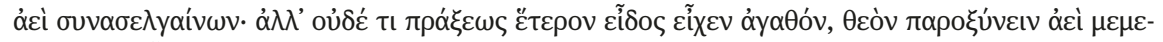

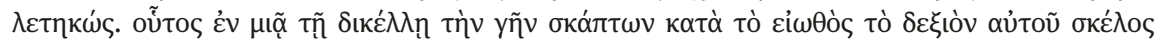

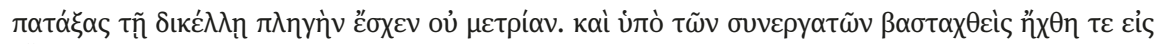

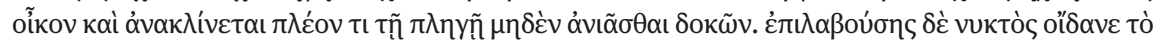

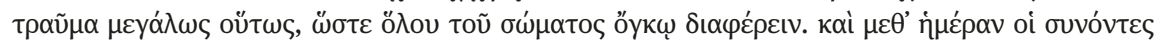

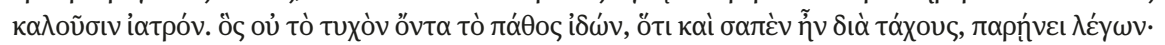

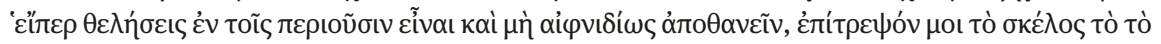

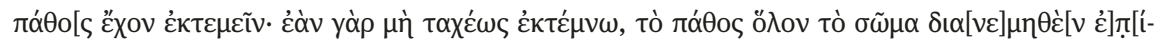

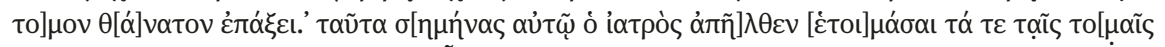

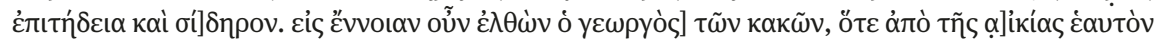

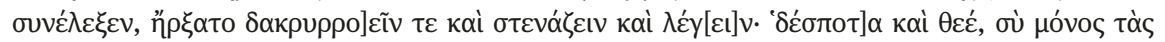

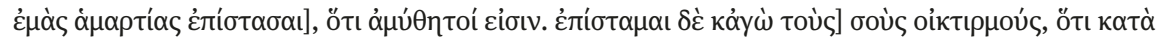

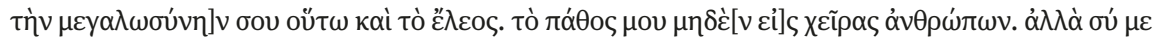

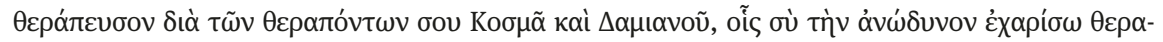

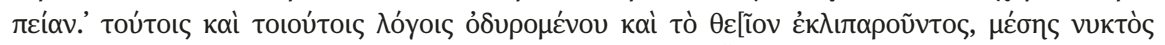

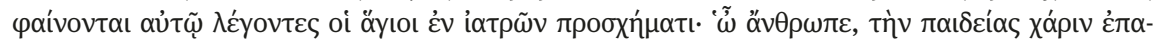

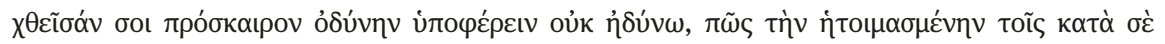

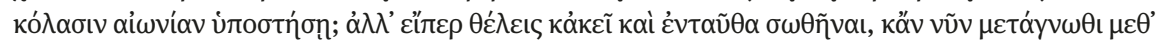

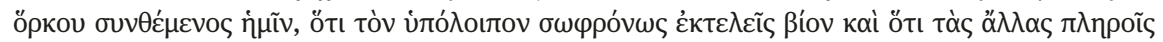

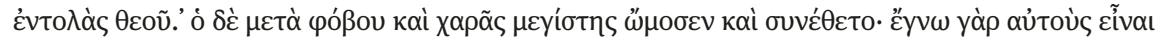

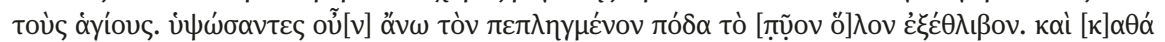

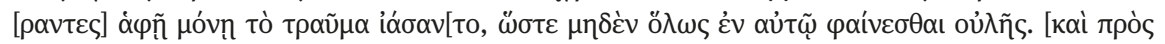

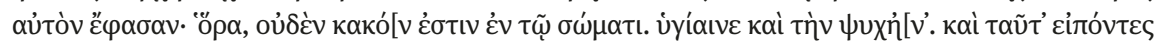

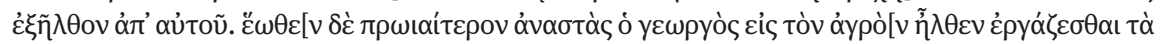

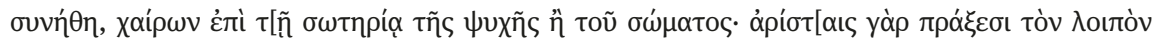

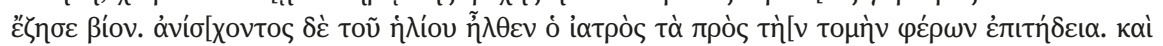


Man erkennt leicht, dass es in dieser Geschichte um christliche Inkubation geht, also eine über einen nächtlichen Traum im Heiligtum vermittelte Heilung, der ein Ritual der Reinigung und Bekehrung vorausgeht, ganz wie in Epidaurus und in anderen paganen Heiligtümern des Asclepius oder Apollon, in denen Inkubation geübt wurde. Allerdings treten hier an die Stelle der paganen Gottheiten zwei christliche Arztheilige namens Cosmas und Damian, die den Titel ávápyupo (anárgyroi) tragen, zu Deutsch: Die, „die (im Unterschied zu den anderen Ärzten) gratis heilen“. Ob die vielfältigen spätantiken Erzählungen über das Wirken der beiden Heiligen wirklich auf das heilende Handeln historischer Figuren zurückgehen (die dann vermutlich aus Syrien stammten) oder ob es sich - wie unser Akademiemitglied, der Berliner klassische Philologe Ludwig Deubner, annahm um eine ahistorische Kontrafaktur der Dioskuren Castor und Pollux handelt, kann für unsere Zusammenhänge offen bleiben (Deubner 1907, S. 50 -52). Bemerkenswerterweise ist unsere Geschichte in der besten griechischen, aus byzantinischer Zeit stammenden Londoner Handschrift der Vita ${ }^{3}$ auch intern datiert, weil nach drei Wundergeschichten aus der angeblichen Lebenszeit der beiden Ärzte „auch das, was danach bei uns durch sie geschehen ist“, erzählt werden soll, also eine Begebenheit, die die Autoren der in der Londoner Handschrift enthaltenen Sammlung irgendwann zwischen der angeblichen Lebenszeit der beiden heiligen Protagonisten (Ende des 3. oder zu Beginn des 4. Jahrhunderts) und ihrer eigenen Zeit datieren. Letztere ist schwer $\mathrm{zu}$ bestimmen, vielleicht entstand eine erste Version im 5. oder 6. Jahrhundert. In jedem Fall kommen wir auf eine (allerdings eher unpräzise) Datierung für unsere Geschichte in der spätantiken Sammlung auf die justinianische und nachjustinianische Zeit. Die Redewendung „bei uns“ bezieht sich vermutlich auf die an der heutigen syrischtürkischen Grenze gelegene antike Stadt Cyrrhus, bei der der bislang nicht identifizierte Ort Pheremma lag, in dem die Heiligen Cosmas und Damian vielleicht erstmals verehrt wurden (vgl. L 1, p. 9,27 R.). Möglicherweise ist aber auch an das Heiligtum der beiden Ärzte Cosmas und Damian in einem Vorort der Reichs-

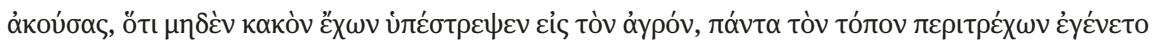

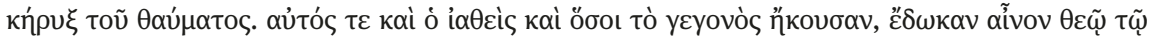

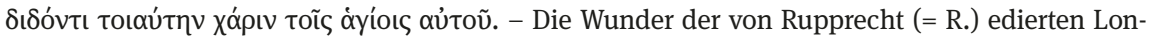
doner Handschrift werden sowohl im Haupttext als auch in den Anmerkungen als „L.“ (hier also: L 4), die bei Deubner (vgl. dazu folgende Anmerkung) aus verschiedenen Handschriften edierten als „D.“ zitiert.

3 British Library BM Add 37534 P 7944, saec. X, Fundort bei Edfu. Es handelt sich um Sondergut (Rupprecht 1935, Tabelle XIV), das sich nicht in den von Deubner edierten Handschriften findet (vgl. auch Bruns 2004). 
hauptstadt Konstantinopel gedacht, ${ }^{4}$ das unter Kaiser Justinian prächtig umgebaut wurde, aber schon vorher existierte (vermutlich wurde es unter der Regierungszeit des Kaisers Theodosius II. gebaut: 408-450 n.Chr.). Der Bauer allerdings, dessen verfaulter Fuß nach unserer Geschichte einer Amputation zum Opfer zu fallen droht, ist zu diesem oder jenem Heiligtum gar nicht in Beziehung gesetzt; die beiden Heiligen scheinen ihm an unbestimmtem Ort zu Hause im Schlaf zu erscheinen und sichern über Nacht nicht nur den Fuß, sondern zugleich auch mit seiner unversehrten Arbeitskraft seine Lebensgrundlagen in der Landwirtschaft. Solche Geschichten wurden allerdings schon in Epidaurus zu Werbezwecken erzählt und diese schlichte Tatsache mag die Verbindung zwischen unserem Text und dem Heiligtum in der syrischen Provinz oder in der Reichshauptstadt dargestellt haben. Die hinter aller Werbung liegende Konkurrenz zwischen Ärzten und ihren Heilweisen ist auch deutlich in unserem Text zu spüren, der einen Vertreter einer schmerzärmeren, dazu erfolgreicheren Therapie gegen einen Arzt stellt, der für seine schmerzhaftere Therapie zudem auch noch länger braucht. Aber das entspricht dem Genre und findet sich immer wieder in den Geschichten der ebenso kostengünstig wie effektiv und schmerzfrei heilenden Dioskuren Cosmas und Damian. Die Frage, ob eher diese Werbung oder tatsächliche Heilerfolge zum Aufstieg des Christentums in der Antike beigetragen haben, wie Hector Avalos nachzuweisen versucht hat (Avalos 1999), ist im Einzelnen schwer zu entscheiden.

Unsere Geschichte vom Bauern mit dem verfaulten Fuß ist natürlich in vielfacher Hinsicht eine für antikes christliches Menschen- und Gesundheitsbild wie den Zusammenhang beider Bilder typische Geschichte. Eine dramatisch geschilderte, lebensgefährliche Erkrankung wird von vornherein auf einen bestimmten Lebenswandel zurückgeführt und darauf, dass Gott durch diesen

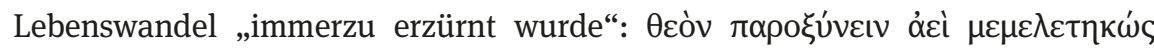
$(15,12$ f. R.). Aber in Wahrheit arbeitet der Text nicht mit so schlichten Kausalitäten, wie es zunächst scheint. Der Bauer wird nicht etwa vom erzürnten Gott mit Krankheit geschlagen, sondern er verletzte sich mit einer Hacke bei der Feldarbeit sein rechtes Bein „und fügte sich dadurch eine tiefe Wunde zu“, wie es wörtlich heißt. Neben der quasi nüchtern volksmedizinisch präsentierten Krankheitsgeschichte läuft zunächst noch ein gleichsam separater Erzählstrang, in dem ein sexuell deviant von den herrschenden christlichen Moralvorstellungen lebender Landwirt aufgrund einer Krankheit zur Einsicht über die (jedenfalls in damaligen Augen bestehende) Verwerflichkeit seines Lebenswandels kommt und diese

4 Das Kosmidion in Blachernai wird jedenfalls in L 7 = D 33 (p. 23,1f. R.) erwähnt, ein Heiligtum am Ort der Bestattung in Syrien am Beginn von L 2 (p. 10,6f. R.). 
Einsicht in Klage und Gebet vor Gott trägt. Der Landwirt appelliert an Gottes Er-

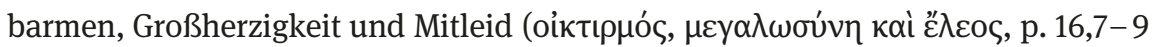
R.). Verknüpft sind beide Stränge durch den Ausdruck „Gnade der schmerzlosen Heilung“ ( eine äußerst schmerzhafte Therapie, die Amputation (Leven 2005), zu vermeiden. Erst die Heiligen Cosmas und Damian, die zur Nacht erscheinen, machen deutlich, dass beide Erzählstränge zusammengehören, weil sie von einem „Dir der

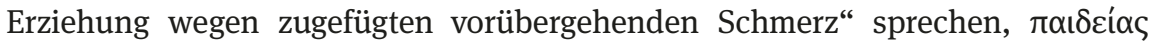

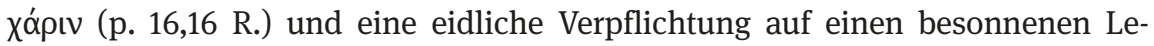
benswandel ( $\sigma \omega \varphi \rho o ́ v \omega \varsigma$, p. 16,22f.) als Voraussetzung der Heilung einfordern und auch erhalten. Die Therapie der Heiligen Cosmas und Damian wird dann wieder ganz schlicht medizinisch geschildert: Eiter wird herausgedrückt, die Wunde gereinigt und nur der Wundverschluss durch Berührung anstelle eines heilenden Pflasters zeigt, dass es sich um besondere Ärzte handelt und es hier um die

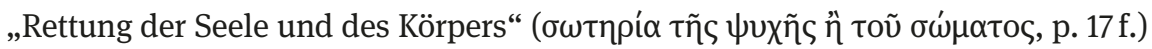
ging. Die körperliche Heilung ist ein Zeichen, das dem Bauern hilft, die Auffor-

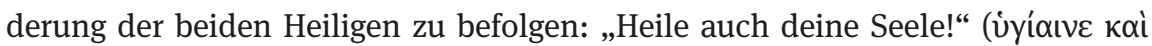

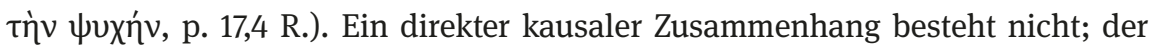
Bauer verbrachte vielmehr aus eigenem Antrieb, aus „Furcht und großer Freude“ sein Leben hinfort mit ethisch vorbildlichen Handlungen. Am Ende steht - wie in der Kirche am Ende eines Gottesdienstes oder einer gottesdienstlichen Handlung - der liturgische Dank der versammelten Gemeinde: Der durch die Heilung im Schlaf arbeitslose Arzt mit dem Amputationswerkzeug, der Geheilte, aber auch alle, die vom Geschehen gehört hatten, „lobten Gott, der seinen Heiligen eine solche Gnade gewährt“ - mit anderen Worten: Die heilenden Fähigkeiten der beiden Ärzte Cosmas und Damian sind durch einen Gott vermittelte Fähigkeit zur Heilung. Ähnliches steht übrigens im Hintergrund eines berühmten Textes des kaiserzeitlichen Mediziners und Philosophen Galen, den man einmal sein ,philosophisches Testament“ (Nutton 1987, S. 27; zum Abschnitt auch Tieleman 2013, S. 103-104) genannt hat: „Der Gott, der in meiner Heimatstadt Pergamon verehrt wird, erwies seine Kraft und Vorsehung vielfach, aber besonders dadurch, dass er mich einmal von einer Krankheit heilte“. ${ }^{5}$ Aus einem anderen Texts Galens wissen wir sogar, dass der (wie er selbst sagt) „Diener des Gottes (sc. Asclepius)“

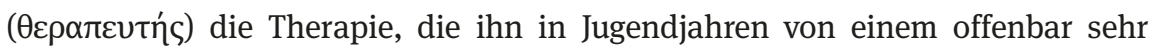

5 Galen, De prop. plac. 2 (Nutton 1987, S. 38 = CMG 5/3,2, 56,19f.). Vgl. den griechischen Text

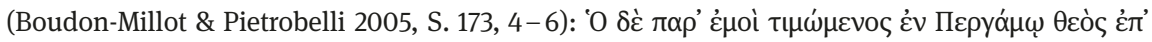

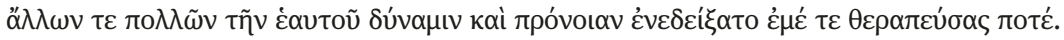


schlimmen Abszess heilte, im Traum empfangen hat. ${ }^{6}$ Auch Galen bringt also eine

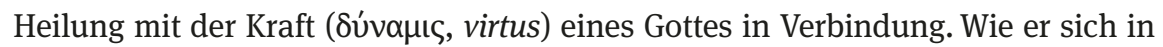
diesem medizinischen Fall die Verteilung seiner Heilung auf einen göttlichen und einen menschlichen, ärztlichen Agenten und die Kräfte seines eigenen Körpers vorstellte, sagt er selbst ebenso wenig wie unser Text aus der Vita der Heiligen Cosmas und Damian.

Unsere Erzählung ist, wie auch explizit festgestellt wird, die Geschichte einer doppelten Heilung, einer Heilung von Körper und Seele. Beide (wenn ich so abgekürzt formulieren darf) Aspekte des Menschen werden dabei nicht strikt getrennt: Der schwer kranke Bauer überdenkt sein Leben und dabei „begann er zu weinen und zu seufzen“, wie es in unserem Text heißt. Er schließt unter Eid mit den Heiligen einen Pakt, hinfort besser zu leben „mit Furcht und größter Freude“ und arbeitet auch am Morgen nach der Heilung mit großer Freude wieder auf dem Feld. Natürlich sind diese Emotionen konstruierter hagiographischer Diskurs und keine psychologische Feinzeichnung, wie schon die biblische Anspielung in der Formulierung „mit Furcht und großer Freude“ zeigt - mit diesen Begriffen werden die beiden Frauen charakterisiert, die nach dem Zeugnis des Matthäusevangeliums vom Grab weglaufen, um die Worte des Engels über die Auferstehung Jesu den Jüngern mitzuteilen (Matthäus 28,8). Aber auch die seelische Heilung ist von Emotionen und Affekten begleitet, präziser: von der Veränderung von Emotionen und der Gegenkonditionierung von Affekten durch heilige Menschen im Traum. Bei diesen Veränderungen und Gegenkonditionierungen hilft, dass das Welt- wie Menschenbild unseres Textes klar dual strukturiert ist (wie es übrigens das Weltund Menschenbild antiker Christenmenschen überhaupt). So wie der Dual von Krankheit und Heilung unsere Geschichte rahmt, prägt sie der Dual von beständigem „unzüchtigem Leben“ und allzeit vorbildlichen Handlungen. Zwischenstufen kommen allenfalls vor, wenn zum Zwecke der Dramatisierung sich die Wunde entzündet, eine große Eiterbeule bildet und Blutvergiftung droht.

Reinhart Koselleck hat solche klar konturierten Duale, bei denen zudem deutlich ist, was davon ein Autor präferiert und seinem Publikum ans Herz legt, „asymmetrische Gegenbegriffe“ genannt. Er hat in einem sehr eindrücklichen Aufsatz darauf aufmerksam gemacht, dass solche ,asymmetrische Gegenbegriffe“ nicht nur die Begriffswelt, sondern die Wahrnehmung von Welt und Mensch strukturieren (Koselleck 1995). Sie folgen dem Freund-Feind-Schema von „wir hier“ und „ihr da“ und exakt diesem, leicht von einem Text auf einen anderen Text

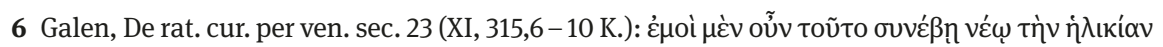

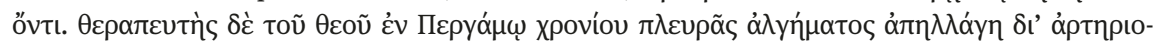

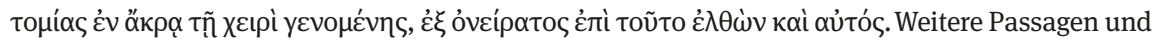
Details bei: Schlange-Schöningen 2003, S. 226. 
und von einer Situation auf eine andere Situation übertragbaren Schema folgt auch unser Text aus der Vita der Heiligen Cosmas und Damian. Auf diese Weise werden Duale konstruiert, denen sich niemand mehr entziehen kann, es sei denn, die asymmetrischen Duale schlagen irgendwann in Symmetrien um. Solche Duale sind aber auch ein Kennzeichen eines eher laienhaften Blicks auf die Wirklichkeit, der noch nicht durch Professionalisierung einer Wissenschaft - beispielsweise der Medizin - in mehr oder weniger gradierte Skalen abgestufter Graubereiche überführt worden ist. Diese Alternative zwischen asymmetrischen Gegenbegriffen und eher skalenartigen Modellen könnte man nun an den Vorstellungen von Gesundheit in der Antike und in unserem Text näher beschreiben.

Zunächst aber zum Menschenbild: Das Menschenbild, das in unserem Text zum Ausdruck kommt, impliziert einen engeren Zusammenhang von körperlichen und seelischen Befindlichkeiten; hier wird also Gesundheit nicht nur technisch als Abwesenheit von Störung körperlicher Funktionen verstanden. Der Mensch ist frei, sich bestimmten Lebensformen zuzuwenden, und diese Lebensformen haben körperliche und seelische Folgen. Der Mensch ist auch frei, sich von einer selbst gewählten Lebensform wieder abzuwenden; Gott, der ihn geschaffen hat zu ewigem Heil, bietet ihm durch Affekte und Emotionen Gelegenheit zu Verhaltensänderung und Einübung alternativer Verhaltensweisen. Er kann das, weil offensichtlich Lebensformen und Verhaltensänderung mit bestimmten Affekten wie Emotionen zu tun haben. Unser Text nennt diesen Zusammenhang Erziehung, $\pi \alpha ı \varepsilon \varepsilon^{\prime} \alpha$ (paideía). Der Mensch ist also frei und zugleich ein erziehungsbedürftiges Mängelwesen. Erziehung geschieht durch Schmerz - wie es übrigens ja bis weit ins 20. Jahrhundert in vielen Haushalten auch hierzulande noch üblich war. Sexualität muss eingehegt werden und ist latent für die eigene Seele und das Vermögen gefährlich, insbesondere wenn der Trieb mit Prostituierten befriedigt wird. Bekanntlich traf das Christentum in der römischen Kaiserzeit schon auf eine entsprechend disponierte Schamkultur und einen gewissen Puritanismus herrschender Kreise, wie Michel Foucault und Paul Veyne, aber auch Peter Brown und viele andere gezeigt haben. (Aus der Fülle einschlägiger Literatur seien genannt: Brown 1991; Foucault 2019; Gehring \& Markschies 2019; Le Goff \& Truong 2007, S. 53-54.) Insofern dient unser Text mit dem Wunder der Heiligen Cosmas und Damian natürlich nicht nur zu Werbezwecken, sondern auch als didaktisch motivierte Erzählung, die ein bestimmtes Welt- und Menschenbild in Erinnerung bringen und festigen will. Gesundheit ist also weder etwas, was rein den Körper betrifft, noch allein Sache der Ärzte - man kann selbst gesund werden, auch ohne direkte Hilfe der professionellen Medizin. Wir erinnern uns an den einschlägigen

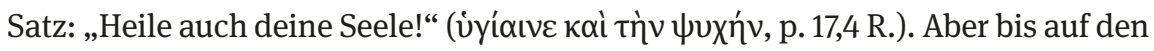
heutigen Tag konstatieren wir ja einen nicht geringen Anteil an Menschen, die 
sich mit oder ohne Anleitung von Fachleuten selbst therapieren und dabei mitunter durchaus erfolgreich sind.

Das Bild von Gesundheit in unserem Text ist - wie mir jedenfalls scheint zunächst einmal stark laienmedizinisch geprägt. Es basiert auf dem grundlegenden Dual - oder nach Koselleck: den asymmetrischen Gegenbegriffen - von Gesundheit und Krankheit, die natürlich innerhalb beider Zustände noch die Steigerung (beispielsweise Steigerung der Krankheit durch Entzündung und Blutvergiftung) kennen. Fachwissenschaftliche Einsichten der Antike wie die Lehre von der Mischung der Körpersäfte spielen hier keinerlei Rolle (Kudlien 1978, S. 907-909). Aber fachwissenschaftliche Theorien über psychosomatische $\mathrm{Zu}$ sammenhänge spiegeln sich natürlich in der Laienmedizin, die sich wiederum in unserem Text, der „Vita ... und der Wunder der Heiligen Gratisheiler Kosmas und Damian“ wiederfinden. Wichtig ist, dass ohne jede Diskussion vorausgesetzt ist, dass körperliche Gesundheit einen Wert darstellt - bestimmte radikale Kreise des spätantiken Mönchtums sahen das durchaus anders und trennten sehr viel mehr zwischen der anzustrebenden Gesundheit der Seele und der Abtötung, ja allmählichen Vernichtung des eigenen Leibes (Markschies 2004). Man wird also sicher für die Antike auch nicht von einem einzigen christlichen Bild von Gesundheit sprechen können; die christlichen Konzepte schwanken zwischen Körperfreudigkeit und Körperhass (Kudlien 1978, S. 939).

So viel zur Antike. Auf den ersten Blick scheint unsere Gegenwart mit dieser Welt der Vormoderne wenig zu tun zu haben. Der römisch-katholische Grazer Pastoraltheologe Rainer Bucher spricht von einem neuzeitlichen Funktionsverlust der Religion im Blick auf die Heilung von Krankheit und Gesundheit - Teil eines umfassenderen Funktionsverlustes von Religion oder jedenfalls eines Verlustes von Alleinzuständigkeit (Bucher 2010, S. 231-232). Die Verlusterfahrung wird inzwischen kompensiert mit neuen Angebotsformen (wenn sie nicht, wie in bestimmten Kreisen des Christentums, geleugnet wird, beispielsweise indem schlicht die klassische Heilweise der antiken Christenheit fortgesetzt wird (oder in charismatischen Zusammenhängen transformiert revitalisiert wird, deren Vielfalt kaum mehr zu überblicken ist). Nur zwei Beispiele: Da gibt es an verschiedenen Orten das Angebot einer „Heilenden Seelsorge“ (z. B. Baumgartner 1990; Müller 2000), die nach eigener Aussage die Spaltung oder Spannung zwischen Heil und Heilung überwinden will und eine Kombination von christlicher Spiritualität mit Anregungen aus der Psychotherapie versucht, da existiert (in München) ein Studiengang „spiritual care“, der akademische Konsequenzen aus der Definition der Weltgesundheitsorganisation von Palliative Care ziehen will, die Behandlung von Schmerzen sowie anderen Problemen körperlicher, psychosozialer und spiritueller Art umfasst (Vgl. Klinikum der Universität München 2020; zur Palliativmedizin vgl. Nationale Akademie der Wissenschaften Leopoldina \& Union der 
deutschen Akademien der Wissenschaften 2015). Immer stärker wird diese ursprünglich auf die Palliativmedizin konzentrierte Forschungsfrage und Ausbildungseinrichtung ,als grundlegende Dimension der Betreuung von der Geburt bis zum Lebensende“ verstanden, in dem „gleichermaßen Potenziale wie auch Risiken von Religiosität und Spiritualität in den Blick“ genommen werden (Klinikum der Universität München 2017).

So plural, wie die Welt inzwischen ist, sind wohl auch die Menschen- und Gesundheitsbilder. Unser Blick in die Antike wollte davor warnen, die historische Entwicklung dieser Bilder allzu schnell mit Dualen wie dem von Vormoderne und Moderne nachzuzeichnen; es gibt mehr Survivals ${ }^{7}$ von antiken Bildern, als uns vermutlich klar und vielleicht auch lieb ist. Gleichzeitig wird aber ebenfalls deutlich, dass es bereits vor der Moderne Aufklärung - oder sagen wir vorsichtiger und in Anknüpfung an ein Projekt der Akademie - Rationalisierung gab und man sich auch in Kreisen medizinischer Laien um präzise Beschreibung von Verantwortlichkeiten und Ursachen bemühte (Vgl. Markschies 2019). Eine Hacke verursacht die lebensbedrohliche Wunde des Bauern, mit dessen Arbeitsunfall in der Perspektive eines spätantiken Heiligenlebens wir begonnen hatten; Gott nutzt lediglich die Schmerzen zur Pädagogik - so, wie man das damals und noch lange danach von fürsorglichen Eltern erwartete. Die Wirklichkeit war also damals schon „vielspältig“ und nicht nur „vielfältig“, wie Trutz Rendtorff einmal schön differenzierte (Rendtorff 1991), sie ist es heute noch viel mehr.

Es spricht manches dafür, den Faktor Religion bei Menschen- und Gesundheitsbildern (mit dem wir uns beschäftigt haben) für ein Kontinuum, aber nicht für ein überwundenes Moment der Vormoderne $\mathrm{zu}$ halten. Und wenn es um „Gesundheit für alle“ und gar um einen holistischen Zugriff auf das große Thema „Gesundheit“ geht, dann kann man sich es erst recht nicht leisten, den Faktor Religion zu dispensieren und in einen naiven Reduktionismus zu verfallen. Bereits ein oberflächlicher Blick in Berliner Praxen und Krankenhäuser zeigt, wie wichtig dieser Faktor im medizinischen Alltag ist - und entsprechend auch in der Ausbildung und theoretischen Reflexion sein sollte. Allerdings darf man ihn natürlich auch nicht überschätzen - wir sahen bei unserer Analyse antiker Texte, dass es immer wieder schon in vormodernen Zeiten eine Autonomie von Gesundheitsbildern gibt, die weder über Korrelations- noch gar Kausalbeziehungen mit ganz bestimmten Menschenbildern verbunden werden können. Ein streng religiöses Menschenbild kann schon in der römischen Kaiserzeit unverbunden neben einem vollkommen säkularen Gesundheitsbild stehen und verschiedene

7 Der Begriff „Survival“ übersetzt den in der Bibliothek Warburg geprägten Begriff „Nachleben“ (Landauer 1984). 
christliche antike Texte wie beispielsweise die spätantiken Wunder der Heiligen Cyrus und Johannes, die der Jerusalemer Patriarch Sophronius Anfang des 7. Jahrhunderts gesammelt hat (Booth 2009, S. 52-63; Fernández Marcos 1975, S. 23 -32), belegen dieses auf den ersten Blick verblüffende Nebeneinander. In der europäischen Neuzeit dürften sich solche für unseren Geschmack eher widersprüchlichen Zusammentreffen eher gesteigert haben. Angesichts des allgemeinen Pluralisierungsschubs, der sich natürlich auch auf Menschen- wie Gesundheitsbilder auswirkt, muss man nicht nur einüben, mit der kaum verbundenen Kopräsenz sehr unterschiedlicher Verständnisse zu leben, sondern sorgfältig über Traditionslinien und andere Beziehungen zwischen diesen Verständnissen nachdenken. Wenn solche sorgfältigen traditionsgeschichtlichen und kompositorischen Analysen ein Teil der holistischen Agenda sind, muss man sich nicht besorgen; wenn sie bisher fehlen, sollte entsprechend nachgearbeitet werden. ${ }^{8}$

\section{Literatur}

Avalos, Hector (1999): Health Care and the Rise of Christianity. Peabody, MA: Hendrickson. Baumgartner, Isidor (1990): Pastoralpsychologie. Einführung in die Praxis heilender Seelsorge. Düsseldorf: Patmos.

Berlin-Brandenburgische Akademie der Wissenschaften (2020): „Mediathek: Verständnis(se) von Gesundheit, Symposium vom 28. Januar 2020“. Berlin-Brandenburgische Akademie der Wissenschaften. https://www.bbaw.de/mediathek/archiv-2020/verstaendnisse-vongesundheit, besucht am 6.12.2020.

Booth, Phil (2009): „Saints and Soteriology in Sophronius Sophista's Miracles of Cyrus and John“. In: Studies in Church History 45, S. 52-63.

Boudon-Millot, Véronique/Pietrobelli, Antoine (2005): „Galien ressuscité. Édition princeps du texte grec du De propriis placitis“. In: Revue des Études Grecques 118, S. 168-213.

Brown, Peter (1991): Die Keuschheit der Engel: Sexuelle Entsagung, Askese und Körperlichkeit am Anfang des Christentums. München: Hanser.

Bruns, Peter (2004): „Die Syrische Kosmas- und Damian-Legende“. In: Rivista di archeologia cristiana 80, S. 195-210.

Bucher, Rainer (2010): „Pastorale Heilungspraktiken. Überlegungen zu ihrer Attraktivität und Problematik“. In: Walter Schaupp/Hans-Walter Ruckenbauer (Hrsg.): Macht Religion gesund? Christliches Heilsangebot und Wellness-Kultur. Innsbruck: Tyrolia, S. 231-245.

Deubner, Ludwig (1907): Kosmas und Damian. Texte und Einleitung. Leipzig \& Berlin: BG Teubner.

Fernández Marcos, Natalio (1975): Los Thaumata de Sofronio: Contribución al estudio de la incubatio cristiana. Madrid: Instituto Antonio de Nebrija.

8 An dieser Stelle greife ich auf frei formulierte Bemerkungen aus meinem Versuch einer Zusammenfassung der Berliner Tagung am 28. Januar 2020 zurück, der in der Mediathek der BBAW zugänglich ist: (Berlin-Brandenburgische Akademie der Wissenschaften 2020). 
Foucault, Michel (2019): „Die Geständnisse des Fleisches“. Sexualität und Wahrheit (Bd. 4), herausgegeben von Frédéric Gros. Berlin: Suhrkamp.

Gehring, Petra/Markschies, Christoph (2019): „Michel Foucault: Wie der Sex zur Sünde wurde“. Zeit Online. https://www.zeit.de/2019/27/michel-foucault-sexualitaet-und-wahrheitvierter-band, besucht am 6.12.2020.

Klinikum der Universität München (2017): „Stiftungsprofessur für Spiritual Care mit Prof. Dr. Constantin Klein wiederbesetzt“. https://www.med.uni-muenchen.de/aktuell/2017/ stiftungsprofessur/index.html, besucht am 6.12.2020.

Klinikum der Universität München (2020): „Professur für Spiritual Care“. Klinikum der Universität München. http://www.klinikum.uni-muenchen.de/Klinik-und-Poliklinik-fuerPalliativmedizin/de/professur-fuer-spiritual-care/index.html, besucht am 6.12.2020.

Koselleck, Reinhart (1995): „Zur historisch-politischen Semantik asymmetrischer Gegenbegriffe“. In: Reinhart Koselleck (Hrsg.): Vergangene Zukunft. Zur Semantik geschichtlicher Zeiten. Frankfurt am Main: Suhrkamp, S. 211-259.

Kudlien, Friedolf (1978): „Gesundheit“. In: Reallexikon für Antike und Christentum: Sachwörterbuch zur Auseinandersetzung des Christentums mit der antiken Welt, Bd. 10. Stuttgart: Hiersemann, S. 902-945.

Landauer, Carl Hollis (1984): „The Survival of Antiquity. The German Years of the Warburg Institute“ [PhD., Yale University]. New Haven.

Le Goff, Jacques/Truong, Nicolas (2007): Die Geschichte des Körpers im Mittelalter. Stuttgart: Klett-Cotta.

Leven, Karl-Heinz (2005): „Amputation“. In: Antike Medizin. Ein Lexikon. München: C. H. Beck, S. 38.

Markschies, Christoph (2004): „Körper und Körperlichkeit im antiken Mönchtum“. In: Barbara Feichtinger/Helmut Seng (Hrsg.): Die Christen und der Körper. Aspekte der Körperlichkeit in der christlichen Literatur der Spätantike. München: K. G. Saur, S. 189-212.

Markschies, Christoph (2006a): „Einführung: Heil und Heilung in der Spätantike“. In: Humboldt-Universität zu Berlin/Berliner Medizinhistorisches Museum der Charité (Hrsg.): Wunderheilungen in der Antike. Von Asklepios zu Felix Medicus. Begleitheft zur gleichnamigen Ausstellung vom 10. November 2006 - 11. März 2007. Oberhausen: Athena, S. 17-23.

Markschies, Christoph (2006b): „Gesund werden im Schlaf. Einige Rezepte aus der Antike“. In: Berlin-Brandenburgische Akademie der Wissenschaften. Berichte und Abhandlungen 12, S. $187-216$.

Markschies, Christoph (2008): „Heil und Heilung: Transformationen antiker Heilkulte“. Theologische Fakultät der Humboldt-Universität zu Berlin. https://www.antikeschristentum.de/apokryphen8, besucht am 16.12.2020.

Markschies, Christoph (2019): „Introduction“. In: Yohanan Friedmann/Christoph Markschies (Hrsg.): Rationalization in Religions. Judaism, Christianity and Islam. Berlin \& Boston: De Gruyter, S. 1-4.

Müller, Wunibald (2000): Heilende Seelsorge. Mainz: Matthias Grünewald.

Nationale Akademie der Wissenschaften Leopoldina/Union der deutschen Akademien der Wissenschaften (2015): Stellungnahme. Palliativversorgung in Deutschland. Perspektiven für Praxis und Forschung. Halle an der Saale: Nationale Akademie der Wissenschaften Leopoldina, https://www.leopoldina.org/uploads/tx_leopublication/2015_ Palliativversorgung_LF_DE.pdf, besucht am 6.12.2020. 
Nutton, Vivian (1987): „Galen’s Philosophical Testament: ,My Own Opinions““. In: Jürgen Wiesner (Hrsg.): Aristoteles. Werk und Wirkung. Bd. 2: Kommentierung, Überlieferung, Nachleben: Paul Moraux zum 65. Geburtstag gewidmet. Berlin \& New York: De Gruyter, S. $27-51$.

Rendtorff, Trutz (1991): Vielspältiges. Protestantische Beiträge zur ethischen Kultur. Stuttgart, Berlin \& Köln: Kohlhammer.

Rupprecht, Ernst (Hrsg.) (1935): Cosmae et Damiani Sanctorum medicorum vitam et miracula e codice Londinensi. Berlin: Junker und Dünnhaupt.

Schlange-Schöningen, Heinrich (2003): Die römische Gesellschaft bei Galen: Biographie und Sozialgeschichte. Berlin \& New York: De Gruyter.

Thumiger, Chiara (2020): „Holism, Parts, Wholes“. In: Chiara Thumiger (Hrsg.): Holism in Ancient Medicine and Its Reception. Leiden \& Boston: Brill, S. 25-46.

Tieleman, Teun (2013): „Miracle and Natural Cause in Galen“. In: Stefan Alkier/Annette Weissenrieder (Hrsg.): Miracles Revisited. New Testament Miracle Stories and their Concepts of Reality. Berlin \& New York: De Gruyter, S. 101-114. 